\title{
ON POLARIZED K3 SURFACES OF GENUS 33
}

\author{
ILYA KARZHEMANOV
}

\begin{abstract}
We prove that the moduli space of smooth primitively polarized K3 surfaces of genus 33 is unirational.
\end{abstract}
\section{INTRODUCTION}

Let $S$ be a K3 surface (i.e. smooth projective simply-connected surface with trivial first Chern class). One may regard such surfaces as two- dimensional counterparts of elliptic curves. In fact, K3 surfaces turn out to be favourably endowed with geometric, arithmetic and group-theoretic properties (cf. [26], [2], 24], [25], 4]).

In the present paper, we study birational geometry of the moduli space of primitively polarized K3 surfaces over C. Namely, we consider the pairs $(S, L)$, where $S$ is a complex K3 surface and $L$ is an ample divisor on $S(L$ is called polarization of $S$ ) corresponding to some primitive vector in the Picard lattice $\operatorname{Pic}(S)$. Once the integer $g:=\left(L^{2}\right) / 2+1$ (called the genus of $\left.(S, L)\right)$ is fixed, the pairs $(S, L)$, when considered up to isomorphisms preserving $L$, are parameterized by the moduli space $\mathcal{K}_{g}$.

Recall that $\mathcal{K}_{g}$ is a quasi-projective algebraic variety (see [1, 27]). In particular, one may study such basic questions of birational geometry for $\mathcal{K}_{g}$ as rationality, unirationality, rational connectedness, Kodaira dimension estimate, etc.

S. Mukai's vector bundle method, developed in order to classify higher-dimensional Fano manifolds of Picard number 1 and coindex 3 (see [15], [18), allowed him to prove unirationality of $\mathcal{K}_{g}$ for $g \in\{2, \ldots, 10,12,13,18,20\}$ (see [17, 21], [16, 22], [19]). At the same time, variety $\mathcal{K}_{g}$ turned out to be non- unirational for $g \geqslant 41$, with $g \neq 42,45,46$ and 48 (see [7, [13], [14).

In the present paper, applying the methods (mainly) from [15] and [19, we prove the following:

Theorem 1.1. The moduli space $\mathcal{K}_{33}$ is unirational.

Let us briefly outline the proof of Theorem 1.1. It follows from [12, Corollary 1.5] (cf. Proposition 2.9 below) that there exists a $\mathrm{K} 3$ surface $S$ such that $\operatorname{Pic}(S)$ is generated by some very ample divisor $H$ and a $(-2)$ - curve $C$, satisfying $\left(H^{2}\right)=70$ and $H \cdot C=2$. Thus $(S, H)$ is a primitively polarized K3 surface of genus 36 . (Note that all such $(S, H)$ form a hypersurface $\mathcal{K}_{36}^{\mathrm{R}}$ in $\mathcal{K}_{36}$.) Furthermore, the divisors $H-k C, 1 \leqslant k \leqslant 4$, also provide primitive polarizations on $S$ (see Lemma 2.10 and Remark 2.11), so that the surfaces $(S, H-k C$ ) are $B N$ general (see Definition 2.2. Lemma 2.12 and Remark 2.13). In particular, according to S. Mukai there exists a rigid vector bundle $E_{3}$ on $S$ of rank 3, such that the first Chern class $c_{1}\left(E_{3}\right)$ is equal to $H-4 C$ and $\operatorname{dim} H^{0}\left(S, E_{3}\right)=7$ (see Theorem - definition 2.6). This $E_{3}$ is unique and determines a morphism $\Phi_{E_{3}}: S \longrightarrow G(3,7)$ into the Grassmannian $G(3,7) \subset \mathbb{P}\left(\bigwedge^{3} \mathbb{C}^{7}\right)$ (cf. Remark 2.5). Moreover, $\Phi_{E_{3}}$ coincides with embedding $S \hookrightarrow \mathbb{P}^{12}$ given by the linear system $|H-4 C|$, and the surface $S=\Phi_{E_{3}}(S) \subset G(3,7) \cap \mathbb{P}^{12}$ can be described by explicit equations on $G(3,7)$ (see Theorem 2.14). In fact, one may run these arguments for any (BN) general polarized K3 surface $\left(S_{22}, L_{22}\right)$ of genus 12 , which implies that the moduli space $\mathcal{K}_{12}$ is unirational (see Remark 2.15).

Further, vector bundle $E_{3} \otimes \mathcal{O}_{S}(C)$ turns out to be rigid of rank 3 as well, satisfying $c_{1}\left(E_{3}\right) \otimes \mathcal{O}_{S}(C)=H-C$ and $\operatorname{dim} H^{0}\left(S, E_{3} \otimes \mathcal{O}_{S}(C)\right)=14$ (see Lemma 3.2). Then again we get a morphism $\Phi_{E_{3} \otimes \mathcal{O}_{S}(C)}: S \longrightarrow G(3,14) \subset$ $\mathbb{P}\left(\bigwedge^{3} \mathbb{C}^{14}\right)$ which is the embedding $S \hookrightarrow \mathbb{P}^{33}$ given by $|H-C|$. Working a bit more with the space $H^{0}\left(S, E_{3} \otimes \mathcal{O}_{S}(C)\right)$ (cf. Proposition 3.4 and Corollary [3.7) we find two projective subspaces, $\Pi$ and $\Lambda$, in $\mathbb{P}\left(\bigwedge^{3} \mathbb{C}^{14}\right)$ (see $\mathbf{3 . 8}$ in Section 3 for their construction) such that the following holds (cf. Lemma 3.10):

MS 2010 classification: 14J28, 14J15, 14M20.

Key words: K3 surface, moduli space, unirationality. 
Theorem 1.2. The surface $S=\Phi_{E_{3} \otimes \mathcal{O}_{S}(C)}(S)$ coincides with Zariski closure of the locus $\widehat{S} \backslash \Pi$ in

$$
\widehat{S}:=G(3,14) \cap \Lambda \cap\left(\lambda=\sigma_{1}=\sigma_{2}=\sigma_{3}=0\right)
$$

for some global sections $\lambda \in H^{0}\left(G(3,14), \bigwedge^{3} \mathcal{E}_{14}\right) \simeq \bigwedge^{3} \mathbb{C}^{14}$ and $\sigma_{i} \in H^{0}\left(G(3,14), \bigwedge^{2} \mathcal{E}_{14}\right) \simeq \bigwedge^{2} \mathbb{C}^{14}$ of the universal bundle $\mathcal{E}_{14}$ on $G(3,14)$.

Now, any general polarized $\mathrm{K} 3$ surface $\left(S_{64}, L_{64}\right)$ of genus 33 can be embedded into $G(3,14)$ the same way as $S$ above, and represented in $G(3,14)$ in the form similar to that in Theorem 1.2 (see $\mathbf{4 . 1}$ and Lemma 4.7 for details). The latter allows one, by using the preceding results, to construct a birational map $\mathcal{K}_{33} \rightarrow \mathcal{K}_{12}$ (see Lemma 4.8 and Proposition 4.9) and to finish the proof of Theorem 1.1

Finally, the paper concludes with Remark 4.10, where we sketch a related approach to prove unirationality of $\mathcal{K}_{36}$.

Acknowledgments. I would like to thank A. Lopez, Yu. G. Prokhorov, and F. Viviani for fruitful conversations. I am also grateful to anonymous referee for helpful remarks. The work was supported by World Premier International Research Initiative (WPI), MEXT, Japan, Grant-in-Aid for Scientific Research (26887009) from Japan Mathematical Society (Kakenhi), and by the Project TROPGEO of the European Research Council..

\section{Preliminaries}

2.1. In the present section, we recall some notions and facts about (primitively) polarized K3 surfaces, which will be used through the rest of the paper (see also [6], 8] and [9] for other standard notation, notions and facts employed below). We also establish several auxiliary results. The ground field will be $\mathbb{C}$.

Definition 2.2 (see [19, Definition 3.8]). A polarized K3 surface $(\mathfrak{S}, L)$ of genus $g$ is called $B N$ general if $h^{0}\left(\mathfrak{S}, L_{1}\right) h^{0}\left(\mathfrak{S}, L_{2}\right)<g+1$ for all non - trivial line bundles $L_{1}, L_{2} \in \operatorname{Pic}(\mathfrak{S})$ such that $L=L_{1}+L_{2}$.

Example 2.3. Generic point in the moduli space $\mathcal{K}_{g}$ corresponds to a BN general K3. All BN general K3 surfaces of genus $g$ form a Zariski open subset in $\mathcal{K}_{g}$.

Definition 2.4. Let $W$ be a smooth projective variety and $E$ a vector bundle on $W$. Then $E$ is said to be generated by global sections if the natural homomorphism of $\mathcal{O}_{W}$ - modules $e v_{E}: H^{0}(W, E) \otimes \mathcal{O}_{W} \longrightarrow E$ is surjective (one identifies $E$ with its sheaf of sections as usual).

Remark 2.5 (cf. [15, Section 2]). In the setting of Definition 2.4 if $E$ is generated by global sections, then one gets a natural morphism $\Phi_{E}: W \longrightarrow G(r, N)$. Here $r:=\operatorname{rank}(E), N:=h^{0}(W, E)$, and $G(r, N)\left(\right.$ or $\left(G\left(r, \mathbb{C}^{N}\right)\right)$ is the Grassmannian of $r$-dimensional linear subspaces in $\mathbb{C}^{N}$. Morphism $\Phi_{E}$ sends each $x \in W$ to the subspace $E_{x}^{\vee} \subset H^{0}(W, E)^{\vee}\left(E_{x}^{\vee}\right.$ is the dual to the fiber $\left.E_{x} \subset E\right)$. In particular, we have the equality $E=\Phi_{E}^{*}(\mathcal{E})$ for the universal vector bundle $\mathcal{E}$ on $G(r, N)$, so that $H^{0}(W, E)=H^{0}(G(r, N), \mathcal{E})$. Furthermore, if the natural homomorphism $\bigwedge^{r} H^{0}(W, E) \longrightarrow H^{0}\left(W, \bigwedge^{r} E\right)$ induced by the $r$ - th exterior power of $e v_{E}$ is surjective, then $\Phi_{E}$ coincides with embedding $\Phi_{\left|c_{1}(E)\right|}: W \hookrightarrow \mathbb{P}:=\mathbb{P}\left(H^{0}\left(W, c_{1}(E)\right)^{\vee}\right)$ given by the linear system $\left|c_{1}(E)\right|$. More precisely, the diagram

$$
\begin{array}{rll}
\Phi_{E}: W & \longrightarrow G(r, N) \cap \mathbb{P} & \subset G(r, N) \\
\cap & \hookrightarrow & \mathbb{P}\left(\bigwedge^{r} H^{0}(W, E)^{\vee}\right)
\end{array}
$$

commutes, where $G(r, N) \subset \mathbb{P}\left(\bigwedge^{r} H^{0}(W, E)^{\vee}\right)$ is embedded via Plücker.

Theorem - definition 2.6 (see [19], 15, [20, 23]). Let $(\mathfrak{S}, L)$ be a polarized K3 surface of genus $g$. Assume that $(\mathfrak{S}, L)$ is BN general. Then for every pair of integers $(r, s)$, with $g=r s$, there exists a (Gieseker) stable vector bundle $E_{r}$ on $\mathfrak{S}$ of rank $r$, such that the following holds:

(1) $c_{1}\left(E_{r}\right)=L$;

(2) $H^{i}\left(\mathfrak{S}, E_{r}\right)=0$ for all $i>0$ and $h^{0}\left(\mathfrak{S}, E_{r}\right)=r+s$; 
(3) $E_{r}$ is generated by global sections and the natural homomorphism $\bigwedge^{r} H^{0}\left(\mathfrak{S}, E_{r}\right) \longrightarrow H^{0}\left(\mathfrak{S}, \bigwedge^{r} E_{r}\right)=$ $H^{0}(\mathfrak{S}, L)$ is surjective (cf. Remark [2.5);

(4) every stable vector bundle on $\mathfrak{S}$ which satisfies (11) and (2) is isomorphic to $E_{r}$.

$E_{r}$ is called rigid vector bundle.

Remark 2.7. One may replace (2) in Theorem-definition 2.6 by the condition $\chi\left(\mathfrak{S}, E_{r}\right)=r+s$.

2.8. Let $X$ be the Fano threefold with canonical Gorenstein singularities and anticanonical degree $\left(-K_{X}\right)^{3}=70$ (see [9, [10, 11]). Recall that divisor $-K_{X}$ is very ample and the linear system $\left|-K_{X}\right|$ gives an embedding of $X$ into $\mathbb{P}^{37}$. We also have

$$
\operatorname{Pic}(X)=\mathbb{Z} \cdot K_{X} \quad \text { and } \quad \operatorname{Cl}(X)=\mathbb{Z} \cdot K_{X} \oplus \mathbb{Z} \cdot \widehat{E}
$$

for the quadratic cone $\widehat{E} \subset X \subset \mathbb{P}^{37}$ (see [12, Corollary 3.11]).

The next result was proved in [12]:

Proposition 2.9 (see [12, Corollary 1.5]). Generic element $S \in\left|-K_{X}\right|$ is a K3 surface such that the lattice $\operatorname{Pic}(S)$ is spanned by some very ample divisor $H \sim-\left.K_{X}\right|_{S}$ and a $(-2)$ - curve $C:=\left.\widehat{E}\right|_{S}$. Furthermore, we have $\left(H^{2}\right)=70, H \cdot C=2$ and the pairs $(S, H)$ form a unirational hypersurface $\mathcal{K}_{36}^{\mathrm{R}} \subset \mathcal{K}_{36}$.

Let $S$ be the K3 surface as in Proposition 2.9.

Lemma 2.10. $H-4 C$ is an ample divisor on $S$.

Proof. Let $Z \subset S$ be an irreducible curve such that $(H-4 C) \cdot Z \leqslant 0$. Write

$$
Z=a H+b C
$$

in $\operatorname{Pic}(S)$ for some $a, b \in \mathbb{Z}$. Note that $a>0$, since $Z \neq C$, the linear system $|m(H+C)|$ is basepoint-free for $m \gg 1$ (it provides a contraction of $C$ ) and $(H+C) \cdot Z=72 a$. On the other hand, we have

$$
0 \geqslant(H-4 C) \cdot Z=62 a+10 b,
$$

which implies that $b<-6 a$. But in this case we get

$$
\left(Z^{2}\right)=70 a^{2}+4 a b-2 b^{2} \leqslant-26 a^{2}<-2
$$

- a contradiction.

Hence $(H-4 C) \cdot Z>0$ for every curve $Z \subset S$. Then $H-4 C$ is ample by the Nakai-Moishezon criterion and because of $(H-4 C)^{2}=22$.

Remark 2.11. Using the same arguments as in the proof of Lemma 2.10, one can show that $H-k C, 1 \leq k \leq 3$, is an ample divisor on $S$ as well, which provides a polarization of genus $36-2 k-k^{2} 1$ ) It is also possible to see this via geometric arguments. Namely, let $p_{1}: \mathbb{P}^{37} \rightarrow \mathbb{P}^{34}$ be the linear projection from the plane $\Pi$, passing through the conic $C$. The blowup $f_{1}: Y_{1} \longrightarrow X$ of $C$ resolves indeterminacies of $p_{1}$ on $X$ and gives a morphism $g_{1}: Y_{1} \longrightarrow X_{1}:=p_{1}(X)$. It can be easily checked that $Y_{1}$ is a weak Fano threefold and $X_{1} \subset \mathbb{P}^{34}$ is an anticanonically embedded Fano threefold of genus 33 (cf. the proof of Proposition 6.12 in [10]). Moreover, we obtain $\operatorname{Pic}\left(Y_{1}\right)=\mathbb{Z} \cdot K_{Y_{1}} \oplus \mathbb{Z} \cdot E_{f_{1}}$, where $E_{f_{1}} \simeq \mathbb{F}_{4}$ is the $f_{1}$ - exceptional divisor, and the morphism $g_{1}$ contracts the surface $f_{1 *}^{-1}(\widehat{E})$ to a point. In particular, the singular locus $\operatorname{Sing}\left(X_{1}\right)$ consists of a unique point, $\operatorname{Pic}\left(X_{1}\right)=\mathbb{Z} \cdot K_{X_{1}}$ and $\mathrm{Cl}\left(X_{1}\right)=\mathbb{Z} \cdot K_{X_{1}} \oplus \mathbb{Z} \cdot E^{(1)}$, where $E^{(1)}:=g_{1 *}\left(E_{f_{1}}\right)$. One can prove that $E^{(1)}$ is the cone over a rational normal curve of degree 4 such that $E^{(1)}=X_{1} \cap \mathbb{P}^{5}$. In particular, there exists a rational normal curve $C_{1} \subset X_{1} \backslash \operatorname{Sing}\left(X_{1}\right)$ of degree 4 , with $C_{1}=X_{1} \cap \Pi_{1}$ for some linear space $\Pi_{1} \simeq \mathbb{P}^{4}$. Proceeding with $X_{1}, \Pi_{1}$, etc. the same way as with $X$, $\Pi$, etc. above, we get three more anticanonically embedded Fano threefolds $X_{2} \subset \mathbb{P}^{29}, X_{3} \subset \mathbb{P}^{22}$, $X_{4} \subset \mathbb{P}^{13}$ of genera $28,21,12$, respectively, such that $\operatorname{Sing}\left(X_{k}\right)$ consists of a unique point, $\operatorname{Pic}\left(X_{k}\right)=\mathbb{Z} \cdot K_{X_{k}}$ and $\mathrm{Cl}\left(X_{k}\right)=\mathbb{Z} \cdot K_{X_{k}} \oplus \mathbb{Z} \cdot E^{(k)}$ for all $k$, where $E^{(k)}$ is the cone over a rational normal curve of degree $2+2 k$. By

\footnotetext{
1) It follows from Proposition 2.9 that these $(S, H-k C)$ form hypersurfaces $\mathcal{K}_{36-2 k-k^{2}}^{\mathrm{R}} \subset \mathcal{K}_{36-2 k-k^{2}}$ (birationally) isomorphic to $\mathcal{K}_{36}^{\mathrm{R}}$ for all $k$.
} 
construction, $S$ is isomorphic to a surface $S_{k} \in\left|-K_{X_{k}}\right|, 1 \leqslant k \leqslant 4$. Furthermore, identifying $S$ with $S_{k}$, we find that $-\left.K_{X_{k}}\right|_{S_{k}} \sim H-k C$ is an ample divisor on $S$, which provides a polarization on $S$ of genus $36-2 k-k^{2}$.

Lemma 2.12. The polarized $\mathrm{K} 3$ surface $(S, H-4 C)$ (of genus 12$)$ is $B N$ general.

Proof. Suppose that

$$
H-4 C=L_{1}+L_{2}
$$

for some non-trivial $L_{1}, L_{2} \in \operatorname{Pic}(S)$ (cf. Definition 2.2). One may assume that both $h^{0}\left(S, L_{1}\right), h^{0}\left(S, L_{2}\right)>0$. Write

$$
L_{i}=a_{i} H+b_{i} C
$$

in $\operatorname{Pic}(S)$ for some $a_{i}, b_{i} \in \mathbb{Z}$. Note that $a_{i} \geqslant 0$ (cf. the proof of Lemma 2.10), hence we get $a_{1}=1, a_{2}=0$, say. The latter implies that $b_{2} \neq 0$.

Now, if $b_{2}<0$, then $h^{0}\left(S, L_{2}\right)=0$ and we are done. Finally, if $b_{2}>0$, then $b_{1} \leqslant-5$ and hence

$$
h^{0}\left(S, L_{1}\right) h^{0}\left(S, L_{2}\right)=h^{0}\left(S, H+b_{1} C\right)<h^{0}(S, H-4 C)=13 \text {, }
$$

since $h^{0}\left(S, L_{2}\right)=h^{0}\left(S, b_{2} C\right)=1$.

Remark 2.13. Using the same arguments as in the proof of Lemma 2.12, one can show that the polarized K3 surfaces $(S, H-k C), 0 \leqslant k \leqslant 3$ (cf. Remark 2.11), are also BN general.

Lemmas 2.10, 2.12 and Theorem - definition 2.6 imply that there exists a rigid rank 3 vector bundle $E_{3}$ on $S$, such that $c_{1}\left(E_{3}\right)=H-4 C$ and $h^{0}\left(S, E_{3}\right)=7$. Then from Remark 2.5 we get the morphism $\Phi_{E_{3}}: S \longrightarrow G(3,7) \cap \mathbb{P}^{12} \subset$ $\mathbb{P}\left(\bigwedge^{3} \mathbb{C}^{7}\right)$ which coincides with embedding $\Phi_{|H-4 C|}: S \hookrightarrow \mathbb{P}^{12}$. We also have $E_{3}=\Phi_{E_{3}}^{*}\left(\mathcal{E}_{7}\right)$ for the universal vector bundle $\mathcal{E}_{7}$ on $G(3,7)$.

Let us recall the explicit description of the image $\Phi_{E_{3}}(S)$ :

Theorem 2.14 (see [19, Theorem 5.5]). The surface $S=\Phi_{E_{3}}(S)$ coincides with the locus

$$
G(3,7) \cap(\lambda=0) \cap\left(\sigma_{1}=\sigma_{2}=\sigma_{3}=0\right)
$$

for some global sections

$$
\lambda \in \bigwedge^{3} H^{0}\left(S, E_{3}\right)=H^{0}\left(G(3,7), \bigwedge^{3} \mathcal{E}_{7}\right) \simeq \bigwedge^{3} \mathbb{C}^{7}
$$

and

$$
\sigma_{1}, \sigma_{2}, \sigma_{3} \in \bigwedge^{2} H^{0}\left(S, E_{3}\right)=H^{0}\left(G(3,7), \bigwedge^{2} \mathcal{E}_{7}\right) \simeq \bigwedge^{2} \mathbb{C}^{7}
$$

Remark 2.15 (see [15, 19, 22]). One may repeat literally the preceding considerations in the case of any BN general polarized K3 surface $\left(S_{22}, L_{22}\right)$ of genus 12 . Namely, $S_{22}$ can be embedded into $G(3,7) \cap \mathbb{P}^{12}$, where it coincides with the locus $G(3,7) \cap(\alpha=0) \cap\left(\tau_{1}=\tau_{2}=\tau_{3}=0\right)$ for some $\alpha \in \bigwedge^{3} \mathbb{C}^{7}$ and $\tau_{1}, \tau_{2}, \tau_{3} \in \bigwedge^{2} \mathbb{C}^{7}$ (so that $\left.\left.\mathcal{O}_{S_{22}}\left(L_{22}\right) \simeq \mathcal{O}_{G(3,7)}(1)\right|_{S_{22}}\right)$. Conversely, any such locus, for generic $\alpha$ and $\tau_{i}$, defines a genus $12 \mathrm{BN}$ general polarized K3 (cf. Example 2.3). This construction also shows that $\left(S_{22}, L_{22}\right)$ is uniquely determined by the $\operatorname{PGL}(7, \mathbb{C})$ - orbits of $\alpha$ and $\tau_{i}$. One then arrives at a birational map between $\mathcal{K}_{12}$ and a $\mathbb{P}^{13}$ - bundle over the orbit space $G\left(3, \bigwedge^{2} \mathbb{C}^{7}\right) / / P G L(3, \mathbb{C})$. From the latter fact it is easy to deduce that variety $\mathcal{K}_{12}$ is unirational.

Remark 2.16. Recall that there exists an isomorphism $\delta: G(3,7) \rightarrow G(4,7)$ induced by the canonical bijection between the projective spaces $\mathbb{P}\left(\bigwedge^{3} \mathbb{C}^{7}\right)$ and $\mathbb{P}\left(\bigwedge^{4} \mathbb{C}^{7}\right)$ (see [3, Ch. III, $\left.\left.\S 11.13\right]\right)$. Then the composition $\Psi:=\delta \circ \Phi_{E_{3}}$ : $S \longrightarrow G(4,7)$ coincides with embedding $\Phi_{|H-4 C|}: S \hookrightarrow \mathbb{P}^{12}$. Let $\mathcal{E}_{7}^{*}$ be the universal vector bundle on $G(4,7)$. Then we have $\Psi=\left.\Phi_{\mathcal{E}_{7}^{*}}\right|_{S}$ by identifying $S$ with $\Psi(S)$. The latter implies that $\left.\mathcal{E}_{7}^{*}\right|_{S}=: E_{4}$ for the rigid rank 4 vector bundle $E_{4}$ on $S$, such that $c_{1}\left(E_{4}\right)=H-4 C$ and $h^{0}\left(S, E_{4}\right)=7$ (cf. Theorem-definition 2.6). Indeed, the inclusion $S=\Psi(S) \subset G(4,7) \cap \mathbb{P}^{12}$ coincides with the morphism $\Phi_{E_{4}}$ (see the construction in Remark 2.5), which 
gives $E_{4}=\left.\mathcal{E}_{7}^{*}\right|_{S}$. Furthermore, it follows from Theorem 2.14 that the surface $S \subset G(4,7) \cap \mathbb{P}^{12}$ coincides with the locus

$$
\left(\lambda^{*}=0\right) \cap\left(\sigma_{1}^{*}=\sigma_{2}^{*}=\sigma_{3}^{*}=0\right)
$$

for some $\lambda^{*} \in H^{0}\left(G(4,7), \bigwedge^{4} \mathcal{E}_{7}^{*}\right)$ and $\sigma_{i}^{*} \in H^{0}\left(G(4,7), \bigwedge^{2} \mathcal{Q}_{7}^{*}\right)$, where $\mathcal{Q}_{7}^{*}$ is the dual of the universal quotient vector bundle on $G(4,7)$. The same applies to any BN general polarized K3 surface $\left(S_{22}, L_{22}\right)$ (cf. Remark 2.15).

2.17. Let us now establish several properties of the vector bundle $E_{3}$ on the K3 surface $S$ introduced in 2.8 ,

Proposition 2.18. $E_{3}$ is $(H-C)$-stable 2)

Proof. Firstly, since $E_{3}$ is stable, it is $(H-4 C)$-semistable (see [5. Ch. 4]). Moreover, $E_{3}$ is actually $(H-4 C)$ stable, for otherwise there exists a coherent subsheaf $F \subseteq E_{3}$ such that $0<\operatorname{rank}(F)<3$ and

which is impossible.

$$
c_{1}(F) \cdot(H-4 C)=\frac{22}{3} \operatorname{rank}(F) \notin \mathbb{Z},
$$

Further, if $E_{3}$ is not $(H-C)$ - stable, then the $(H-4 C)$ - stability of $E_{3}$ implies that there is a cycle $Z=a H+b C$, $a, b \in \mathbb{Z}$, such that

$$
(H-4 C) \cdot Z<0 \leqslant(H-C) \cdot Z
$$

and

$$
-\frac{\operatorname{rank}\left(E_{3}\right)^{2}}{4} B\left(E_{3}\right) \leqslant\left(Z^{2}\right)<0
$$

for the Bogomolov number

(see [5, Ch. 9]). We have:

$$
B\left(E_{3}\right):=2 \operatorname{rank}\left(E_{3}\right) c_{2}\left(E_{3}\right)-\left(\operatorname{rank}\left(E_{3}\right)-1\right) c_{1}\left(E_{3}\right)^{2}
$$

$$
\operatorname{rank}\left(E_{3}\right)=3, \quad c_{1}\left(E_{3}\right)=H-4 C, \quad \chi\left(S, E_{3}\right)=7,
$$

hence $c_{2}\left(E_{3}\right)=10$ by the Riemann - Roch formula, and so $B\left(E_{3}\right)=16$. Then it follows from (2.20) that

$$
-18 \leqslant 35 a^{2}+2 a b-b^{2}=36 a^{2}-c^{2}
$$

for $c:=a-b$. At the same time, (2.19) gives

$$
\frac{36}{5} a-c<0 \leqslant 18 a-c
$$

and thus $a, c>0$. From (2.21) and (2.22) we obtain

$$
-18<36 a^{2}-\left(\frac{36}{5}\right)^{2} a^{2}
$$

or equivalently,

$$
-1<-\frac{22}{25} a^{2}
$$

i. e. $a=1$. Now (2.22) implies that $c \in\{8,9, \ldots, 18\}$. But for such values of $a, c$ we get

$$
-18 \leqslant(6-c)(6+c)<-18
$$

(see (2.21)), which is a contradiction.

Proposition 2.18 is completely proved.

\footnotetext{
2) We employ the terminology from [5, Ch. 4].
} 
Lemma 2.23. $\left.E_{3}\right|_{C}=\mathcal{O}_{\mathbb{P}^{1}}(a) \oplus \mathcal{O}_{\mathbb{P}^{1}}(b) \oplus \mathcal{O}_{\mathbb{P}^{1}}(c)$ for some $a \geqslant b \geqslant c \geqslant 0$.

Proof. Indeed, if $a<0$, say, then $s_{1} \wedge s_{2} \wedge s_{3}=0$ on $C$ for all $s_{1}, s_{2}, s_{3} \in H^{0}\left(S, E_{3}\right)$, which is impossible by construction of embedding $\Phi_{E_{3}}$ (cf. Remark 2.5).

Lemma 2.24. For any two (distinct) generic global sections $s_{1}, s_{2} \in H^{0}\left(S, E_{3}\right)$, the zero locus of the global section $s_{1} \wedge s_{2} \in H^{0}\left(S, \wedge^{2} E_{3}\right)$ is of codimension $\geqslant 2$ on $S$.

Proof. Lemma 2.23 implies that one may assume the zero locus of $s_{1} \wedge s_{2}$ to be of codimension $\geqslant 1$. Suppose $s_{1} \wedge s_{2}=0$ on a curve $Z \subset S$. Note that $s_{1} \wedge s_{2} \neq 0$ everywhere on $C$ by Lemma 2.23 and the results from 21, Section 1]. We get $Z \cdot C=0$ and $Z \sim m(H+C)$ for some $m \in \mathbb{N}$. On the other hand, the condition $s_{1} \wedge s_{2}=0$ on $Z$ implies that $Z \subseteq\left(s_{1} \wedge s_{2} \wedge s^{\prime}=0\right)$ for any $s^{\prime} \in H^{0}\left(S, E_{3}\right)$, i. e. we have $H-4 C-m(H+C) \geqslant 0-$ a contradiction.

Remark 2.25. Running the same arguments as in the proof of Proposition 2.18 and Lemmas 2.23, 2.24, we arrive at similar results for the rigid vector bundle $E_{4}$ on $S$ (see Remark 2.16). Namely, one can show that $E_{4}$ is $H$-stable and for any three generic global sections $s_{1}, s_{2}, s_{3} \in H^{0}\left(S, E_{4}\right)$ the zero locus of the global section $s_{1} \wedge s_{2} \wedge s_{3} \in H^{0}\left(S, \wedge^{3} E_{4}\right)$ is of codimension $\geqslant 2$ on $S$.

\section{Proof of Theorem 1.2}

3.1. Fix $S$ and $E_{3}$ as in Section 2 ,

Lemma 3.2. $E_{3} \otimes \mathcal{O}_{S}(C)$ is a rigid vector bundle of rank 3 and such that

- $c_{1}\left(E_{3} \otimes \mathcal{O}_{S}(C)\right)=H-C$;

- $h^{0}\left(S, E_{3} \otimes \mathcal{O}_{S}(C)\right)=14$.

Proof. The equality $c_{1}\left(E_{3}\right)=H-4 C$ implies that

$$
c_{1}\left(E_{3} \otimes \mathcal{O}_{S}(C)\right)=H-C
$$

for the rank 3 vector bundle $E_{3}$. Consider the exact sequence

$$
0 \longrightarrow \mathcal{O}_{S}\left(E_{3}\right) \longrightarrow \mathcal{O}_{S}\left(E_{3} \otimes \mathcal{O}_{S}(C)\right) \longrightarrow \mathcal{O}_{C}\left(E_{3}(-2)\right) \longrightarrow 0 .
$$

From $\chi\left(S, E_{3}\right)=7$ and the Riemann-Roch formula on $C$ we deduce

$$
\chi\left(S, E_{3} \otimes \mathcal{O}_{S}(E)\right)=\chi\left(S, E_{3}\right)+\chi\left(C, E_{3}(-2)\right)=7+7=14
$$

because $\operatorname{deg}\left(E_{3}(-2)\right)=(H-C) \cdot C=4$ for the rank 3 vector bundle $E_{3}(-2):=\left.\left(E_{3} \otimes \mathcal{O}_{S}(C)\right)\right|_{C}$ on $C \simeq \mathbb{P}^{1}$.

Further, since $E_{3}$ is $(H-C)$-stable (see Proposition 2.18), $E_{3} \otimes \mathcal{O}_{S}(C)$ is also $(H-C)$-stable, and hence $E_{3} \otimes \mathcal{O}_{S}(C)$ is stable (see [5, Ch. 4]). Then, as $(S, H-C)$ is BN general of genus 33 (see Remark 2.13), Theoremdefinition 2.6 and Remark 2.7 complete the proof.

Put $\widetilde{E}_{3}:=E_{3} \otimes \mathcal{O}_{S}(C)$. Lemma 3.2 and Theorem - definition[2.6 imply that the morphism $\Phi_{\widetilde{E}_{3}}: S \longrightarrow G(3,14) \subset$ $\mathbb{P}\left(\bigwedge^{3} \mathbb{C}^{14}\right)$ coincides with embedding $\Phi_{|H-C|}: S \hookrightarrow \mathbb{P}^{33}$. In what follows, we identify $S$ with its image

$$
\Phi_{\widetilde{E}_{3}}(S) \subset G(3,14) \cap \mathbb{P}^{33} \subset \mathbb{P}\left(\bigwedge^{3} \mathbb{C}^{14}\right)
$$

and $\widetilde{E}_{3}$ with $\left.\mathcal{E}_{14}\right|_{S}$, where $\mathcal{E}_{14}$ is the universal vector bundle on $G(3,14)$. 
3.3. Let us find the defining equations for $S \subset G(3,14)$.

Choose a basis $\left\{s_{1}, \ldots, s_{7}\right\}$ in $H^{0}\left(S, E_{3}\right)$ for some global sections $s_{i}$ (to be specified later). Let also $t \in H^{0}\left(S, \mathcal{O}_{S}(C)\right)$ be the unique (up to a $\mathbb{C}^{*}$-multiple) global section. Then $\left\{t s_{1}, \ldots, t s_{7}, \xi_{1}, \ldots, \xi_{7}\right\}$ is a basis in $H^{0}\left(S, \widetilde{E}_{3}\right)$ for some global sections $\xi_{i}$ of $\widetilde{E}_{3}$ (cf. Lemma 3.2).

Proposition 3.4. For every $i$, there exists $\lambda_{i} \in H^{0}\left(S, \wedge^{3} E_{3}\right)$ such that

$$
\xi_{i} \wedge t s_{i 1} \wedge t s_{i 2}=t^{3} \lambda_{i}
$$

with appropriate $s_{i 1}, s_{i 2} \in H^{0}\left(S, E_{3}\right)$ (depending on $i$ ).

Proof. Consider an affine cover $\left\{U_{\alpha}\right\}$ of $S$ such that $\left.E_{3}\right|_{U_{\alpha}}$ is trivial and $t_{\alpha}:=\left.t\right|_{U_{\alpha}} \in \mathcal{O}\left(U_{\alpha}\right)$ for all $\alpha$. Put also $s_{j, \alpha}:=\left.s_{j}\right|_{U_{\alpha}}$ for all $j$. By Lemma 2.24 we can complete $\left\{s_{1, \alpha}, s_{2, \alpha}\right\}$ to a basis of $\left.E_{3}\right|_{U_{\alpha} \backslash \Gamma}$ for each $\alpha$, after choosing $s_{1}, s_{2}$ appropriately. Here $\Gamma$ is a codimension $\geqslant 2$ subset in $S$. Then the construction of embeddings $\Phi_{E_{3}}, \Phi_{\tilde{E}_{3}}$ yields

$$
\left.\xi_{i}\right|_{U_{\alpha} \backslash \Gamma}=f_{1, \alpha} s_{1, \alpha}+f_{2, \alpha} s_{2, \alpha}+f_{\alpha} s_{\alpha}^{\prime}
$$

for all $i, \alpha$ and some $s_{\alpha}^{\prime} \in H^{0}\left(S, E_{3}\right)$. (Here all $f_{j, \alpha}, f_{\alpha} \in \mathcal{O}\left(U_{\alpha}\right)$ depend on $i$, and $s_{\alpha}^{\prime}$ a priori depends on both $i$ and $\alpha$.$) In particular, we have$

$$
\left.\left(\xi_{i} \wedge t s_{1} \wedge t s_{2}\right)\right|_{U_{\alpha} \backslash \Gamma}=f_{\alpha} s_{\alpha}^{\prime} \wedge t_{\alpha} s_{1, \alpha} \wedge t_{\alpha} s_{2, \alpha}=: F_{i, \alpha},
$$

so that the collection $\left\{\left(F_{i, \alpha}, U_{\alpha} \backslash \Gamma\right)\right\}$ defines a global holomorphic section of $\mathcal{O}_{S}(H-C)$ on $S \backslash \Gamma$, hence on $S$.

Lemma 3.6. One can choose $s_{1}=: s_{i 1}$ and $s_{2}=: s_{i 2}$ in such a way that $F_{i, \alpha}=\left.t_{\alpha}^{3} \lambda_{i}\right|_{U_{\alpha}}$ for all $\alpha$ and some $\lambda_{i} \in H^{0}\left(S, \bigwedge^{3} E_{3}\right)$ (not depending on $\alpha$ ).

Proof. We have $s_{\alpha}^{\prime} \wedge t s_{1} \wedge t s_{2} \in H^{0}\left(S, \mathcal{O}_{S}(H-2 C)\right)$. This implies that

$$
f_{\alpha}=\frac{F_{i, \alpha}}{s_{\alpha}^{\prime} \wedge t_{\alpha} s_{1, \alpha} \wedge t_{\alpha} s_{2, \alpha}}=\left.\frac{\xi_{i} \wedge t s_{1} \wedge t s_{2}}{s_{\alpha}^{\prime} \wedge t s_{1} \wedge t s_{2}}\right|_{U_{\alpha}}=t_{\alpha} g_{i}
$$

for some meromorphic function $g_{i} \in \mathbb{C}(S)$ (depending on $\xi_{i}$ ). Let us choose $g_{i}$ to have no poles along $C$.

Note that any global section of $\left.\widetilde{E}_{3}\right|_{C}=E_{3}(-2)$ extends to an appropriate $\xi_{i}$ (apply (2) of Theorem-definition 2.6 to the exact sequence from the proof of Lemma 3.2). Furthermore, since $h^{0}\left(C, E_{3}(-2)\right)=7$, one can see that $b+c \leqslant 6$ and $b \leqslant 4$ in Lemma 2.23, with $c \geqslant 2$ as well by construction of $\Phi_{\widetilde{E}_{3}}$.

Now $s_{1}, s_{2}$, after restricting to $C$, can be brought to either of the forms (same for both $\left.j\right) s_{i j}=(*, *, 0)$ or $s_{i j}=(*, 0, *)$. We may assume w.l.o.g. that $\xi_{i}=(0,0,1)$ and $s_{1}, s_{2}$ are $(*, 0, *)$. This yields $\xi_{i} \wedge s_{1} \wedge s_{2}=0$ on $C \cap U_{\alpha}$ and so $g_{i}$ does not have poles along $C$.

Thus we obtain $\xi_{i} \wedge t s_{1} \wedge t s_{2}=g_{i} t s_{\alpha}^{\prime} \wedge t s_{1} \wedge t s_{2}=t^{3} \lambda_{i}$, with $\lambda_{i}:=g_{i} s_{\alpha}^{\prime} \wedge s_{1} \wedge s_{2} \in H^{0}\left(S, \wedge^{3} E_{3}\right)$, because $t^{3} \lambda_{i}=\xi_{i} \wedge t s_{1} \wedge t s_{2}$ is a regular section of $\widetilde{E}_{3}$ and $\lambda_{i}$ does not have poles along $C$.

\section{Lemma 3.6 proves Proposition 3.4}

Corollary 3.7. For all indices $i$, there exist $\lambda_{i}^{\prime}, \lambda_{i}^{\prime \prime} \in H^{0}\left(S, \bigwedge^{3} E_{3}\right)$ (in addition to $\lambda_{i}$ from Proposition 3.4) such that

$$
\xi_{i} \wedge t s_{i 2} \wedge t s_{i 3}-t^{3} \lambda_{i}^{\prime}=\xi_{i} \wedge t s_{i 3} \wedge t s_{i 1}-t^{3} \lambda_{i}^{\prime \prime}=0
$$

with appropriate linearly independent $s_{i 1}, s_{i 2}, s_{i 3} \in H^{0}\left(S, E_{3}\right)$.

Proof. We retain the notation from the proof of Proposition 3.4. Note that since $\Phi_{E_{3}}(C) \subset \mathbb{P}^{10} \cap G(3,7)$, one may assume that $s_{1} \wedge s_{2} \wedge s_{3}=0$ on $C$, after possibly replacing $S$ by another surface projectively equivalent to it. Then regarding $s_{1}, s_{2}, s_{3}$ as vectors in a 3 - dimensional linear $\mathbb{C}(C)$ - space, we obtain $s_{i j}:=s_{j}, 1 \leqslant j \leqslant 3$, such that $\xi_{i} \wedge s_{i 1} \wedge s_{i 2}=\ldots=\xi_{i} \wedge s_{i 3} \wedge s_{i 1}=0$ on $C \cap U_{\alpha}$. This implies the claim exactly as in the proof of Lemma 3.6. 
Remark 3.8. Given two generic $\xi_{i} \neq \xi_{j}$ as above, we observe that the pairs $\left(s_{i k}, s_{i k^{\prime}}\right)$, all $1 \leq k, k^{\prime} \leq 3$, are distinct from the corresponding pairs $\left(s_{j k}, s_{j k^{\prime}}\right)$. Indeed, the only place where dependence on $i$ for $s_{i 1}, s_{i 2}$ appears is in the proof of Lemma 3.6 (same for $j$ and other $k$ ), when we have put $\xi_{i}=(0,0,1)$. Using the generality of $\xi_{i}, \xi_{j}$, it is then possible to set $\xi_{j}=(1,0,0)$, say, which allows one to take $s_{j 1}, s_{j 2}$ in the form $(*, *, 0)$ for instance. This establishes our assertion.

3.9. Take $\lambda, \sigma_{1}, \sigma_{2}, \sigma_{3}$ as in Theorem 2.14 and write

$$
\lambda=\sum_{1 \leqslant j_{1}, j_{2}, j_{3} \leqslant 7} \alpha_{j_{1}, j_{2}, j_{3}} s_{j_{1}} \wedge s_{j_{2}} \wedge s_{j_{3}}, \quad \sigma_{r}=\sum_{1 \leqslant i, j \leqslant 7} \alpha_{i, j}^{(r)} s_{i} \wedge s_{j}
$$

for some $\alpha_{j_{1}, j_{2}, j_{3}}, \alpha_{i, j}^{(r)} \in \mathbb{C}$. We may assume that

$$
\lambda \in \bigwedge^{3} H^{0}\left(S, E_{3} \otimes \mathcal{O}_{S}(C)\right)=H^{0}\left(G(3,14), \bigwedge^{3} \mathcal{E}_{14}\right) \simeq \bigwedge^{3} \mathbb{C}^{14}
$$

and

$$
\sigma_{1}, \sigma_{2}, \sigma_{3} \in \bigwedge^{2} H^{0}\left(S, E_{3} \otimes \mathcal{O}_{S}(C)\right)=H^{0}\left(G(3,14), \bigwedge^{2} \mathcal{E}_{14}\right) \simeq \bigwedge^{2} \mathbb{C}^{14}
$$

by identifying $\lambda$ and $\sigma_{r}$ with

$$
\sum_{1 \leqslant j_{1}, j_{2}, j_{3} \leqslant 7} \alpha_{j_{1}, j_{2}, j_{3}} t s_{j_{1}} \wedge t s_{j_{2}} \wedge t s_{j_{3}} \quad \text { and } \quad \sum_{1 \leqslant i, j \leqslant 7} \alpha_{i, j}^{(r)} t s_{i} \wedge t s_{j},
$$

respectively.

Let also $\Lambda$ be the linear subspace in $\mathbb{P}\left(\bigwedge^{3} \mathbb{C}^{14}\right)$ given by the equations from Corollary 3.7 for various $1 \leqslant i \leqslant 7$. Then we get

by construction.

$$
S \subseteq G(3,14) \cap \Lambda \cap\left(\lambda=\sigma_{1}=\sigma_{2}=\sigma_{3}=0\right)=: \widehat{S}
$$

Fix $s_{i j}$ as in Corollary 3.7 once and for all. Let $U \subset G(3,14)$ be the open subset on which the sections $t s_{i 1} \wedge t s_{i 2} \wedge t s_{i 3}, 1 \leqslant i \leqslant 7$, do not vanish simultaneously (i. e. $U=G(3,14) \backslash \Pi$ as in Theorem 1.2 ).

Lemma 3.10. The surface $S$ coincides with Zariski closure of the locus $\widehat{S} \cap U$.

Proof. Let us regard the sections $t s_{i}, \xi_{i}, 1 \leqslant i \leqslant 7$, as vector-functions on $\widehat{S} \cap U=: S^{0}$. Then, given $x \in S^{0}$, the construction of $\Lambda$ implies that the components of vectors $\xi_{i}(x)$ are uniquely determined (via "Kramer-type" relations) by the components of $s_{i}(x), 1 \leqslant i \leqslant 7$.

Now, restricting the natural (forgetful) map $\pi: G(3,14) \rightarrow G(3,7)$ (mapping all $\xi_{i}$ to zero) onto $S^{0}$, from the conditions $\lambda(x)=\sigma_{1}(x)=\sigma_{2}(x)=\sigma_{3}(x)=0$ we obtain that $\left.\pi\right|_{S^{0}}: S^{0} \rightarrow \pi\left(S^{0}\right)=S \subset G(3,7)$ is a birational map onto a K3 surface of genus 12 . This implies that $S^{0} \subset S$ is a surface and the result follows.

Lemma 3.10 proves Theorem 1.2

\section{Proof of Theorem 1.1}

4.1. We use the same notation as in Sections 2 and 3 . Let us also fix $t s_{1}, \ldots, t s_{7}$ and $\xi_{1}, \ldots, \xi_{7}$ in what follows as corresponding to a particular embedding $G(3,14) \subset \mathbb{P}\left(\bigwedge^{3} \mathbb{C}^{14}\right)$.

We begin with the next supplementary

Lemma 4.2. The irreducible (and reduced) components of the scheme $G_{\Lambda}:=G(3,14) \cap \Lambda$ are as follows:

- Zariski closure of $G_{\Lambda} \backslash \Pi$;

- the schemes $G_{m}$ of dimension 12 , given (outside the rest of irreducible components of $G_{\Lambda}$ ) by the vanishing of various $m$-tuples, $1 \leqslant m \leqslant 7$, of sections $t s_{i 1} \wedge t s_{i 2} \wedge t s_{i 3}$;

- other irreducible components contained in I, the indeterminacy locus of $\pi: G(3,14) \rightarrow G(3,7)$ (cf. the proof of Lemma 3.10), given by the vanishing of all sections $t s_{i} \wedge t s_{j} \wedge t s_{k}$. 
Proof. The first item is clear because $G_{\Lambda} \backslash \Pi$ is birational to $G(3,7)$. As for the remaining items, we recall that the tuples $\left(t s_{i k}, t s_{i k^{\prime}}\right), 1 \leq k, k^{\prime} \leq 3$, are all distinct for different $\xi_{i}$ (see Remark 3.8).

Further, on the open chart where any two of $t s_{i k} \wedge t s_{i k^{\prime}} \neq 0$, intersection $G_{\Lambda} \cap I$ has dimension 20, being a union of $\left(\mathbb{P}^{1}\right)^{4}$ - bundles $I_{\alpha}$ over some (fixed) determinantal locus $\Gamma \subset G(3,14)$. Now, if $t s_{11} \wedge t s_{12}=t s_{12} \wedge t s_{13}=0$, say, then one gets a union of $\mathbb{P}^{2} \times\left(\mathbb{P}^{1}\right)^{4}$ - bundles over a codimension 6 subset in $\Gamma$. Hence this is a proper closed subset in $G_{\Lambda} \cap I$ and so $G_{\Lambda} \cap I$ does not acquire any other irreducible components, besides $I_{\alpha}$, whenever any two of $t s_{i k} \wedge t s_{i k^{\prime}}$ vanish. (The case when one (resp. two) of $t s_{i j}$ vanishes is similar - one gets a $\mathbb{P}^{2} \times\left(\mathbb{P}^{1}\right)^{4}$ - bundle (resp. $\mathbb{P}^{3} \times\left(\mathbb{P}^{1}\right)^{4}$ - bundle) over a codimension 3 (resp. 6) subset in $\Gamma$.)

Similarly, every $G_{m} \backslash I$ is a $\left(\mathbb{P}^{1}\right)^{m}$ - bundle over a smooth, by generality of $s_{i}$, codimension $m$ linear section $\Lambda_{m}$ of $G(3,7)$. Indeed, this can be seen for $G_{m} \backslash I$ on the open chart where any two of $t s_{i} \wedge t s_{j} \neq 0$, by "solving the linear equations" for $\Lambda$. Now, if again some $t s_{i 1} \wedge t s_{i 2}=t s_{i 2} \wedge t s_{i 3}=0$, then one gets a $\mathbb{P}^{2} \times\left(\mathbb{P}^{1}\right)^{m-1}$ - bundle over a codimension $5+m$ subset in $G(3,7)$. Hence this is a proper subset in $G_{m}$ and so $G_{m}$ does not acquire any irreducible components (out of $I$ ) whenever any two of $t s_{i k} \wedge t s_{i k^{\prime}}$ vanish. (The case when some of $t s_{i j}$ vanish is treated similarly.)

Finally, the loci $G_{\Lambda} \backslash \Pi, G_{m}$ and $G_{\Lambda} \cap I_{\alpha}$ define a stratification on $G_{\Lambda}$ by constructible irreducible subsets whose Zariski closures remain irreducible. Thus all these constitute the components of $G_{\Lambda}$.

Remark 4.3. From (the proof of) Lemma 4.2 we obtain a description of all irreducible components of $\widehat{S}$. Namely, they are the surface $S$, (some of) the 20-dimensional schemes $\widehat{S} \cap I_{\alpha}=G_{\Lambda} \cap I_{\alpha}$ (with $\lambda$ and $\sigma_{i}$ being expressed via polynomials in $t s_{i} \wedge t s_{j} \wedge t s_{k}$ ), or the loci $\widehat{S}_{m}:=\widehat{S} \cap G_{m}$, which are $\left(\mathbb{P}^{1}\right)^{m}$ - bundles over $S \cap \Lambda_{m}$ (for $S$ being identified with $\left.\Phi_{E_{3}}(S) \subset G(3,7)\right)$. In particular, again by generality of $s_{i}$, the schemes $\widehat{S}_{m}$ are all smooth and $m \leqslant 2$. This also shows that $\widehat{S} \backslash I$ is a complete intersection (of pure dimension 2).

4.4. Consider the rigid vector bundle $\widehat{E}_{3}$ on the general K3 surface $\left(S_{64}, L_{64}\right)$ of genus 33 . We have $S_{64}=$ $\Phi_{L_{64}}\left(S_{64}\right) \subset G(3,14) \cap \mathbb{P}^{33}$ and $\widehat{E}_{3}=\left.\mathcal{E}_{14}\right|_{S_{64}}$ by Theorem-definition 2.6. In particular, the pair $\left(S_{64}, \widehat{E}_{3}\right)$ varies in a flat family, specializing to $\left(S, \widetilde{E}_{3}\right)$. Identify $S_{64}$ with the general fiber of the corresponding universal fibration over a base $\mathcal{F}$ and denote by $S_{64} / \mathcal{F}$ the entire family. Similarly, considerations from 3.9 yield a subvariety $\mathcal{F}^{\mathrm{R}} \subset \mathcal{F}$ of codimension 1 (cf. Remark 2.11), so that the induced subfamily $S / \mathcal{F}^{\mathrm{R}} \subset S_{64} / \mathcal{F}$ has $S$ as its general fiber.

The linear projection $\pi: G(3,14) \rightarrow G(3,7)$ induces a rational map

$$
\mathcal{F} \rightarrow\left(\bigwedge^{3} H^{0}\left(S, E_{3}\right) \oplus\left(\bigwedge^{2} H^{0}\left(S, E_{3}\right)\right)^{\oplus 3}\right) \supset \mathcal{F}^{\mathrm{R}}
$$

which we again denote by $\pi$. Note that by construction $\pi\left(\mathcal{F}^{\mathrm{R}}\right)=\mathcal{F}^{\mathrm{R}}$ at the general point.

Lemma 4.5. One has (generically) $\pi(\mathcal{F})=\mathcal{F}^{\mathrm{R}}$. In particular, a typical $\pi$ - fiber is an irreducible curve in $\mathcal{F}$, and $\mathcal{F}^{\mathrm{R}}$ is a $\pi$-section.

Proof. Firstly, the restriction $\left.\pi\right|_{\mathcal{F}_{\mathrm{R}}}$ is smooth because, again by construction, it induces an isomorphism on the tangent space of $\mathcal{F}^{\mathrm{R}}$.

Further, if $\pi(\mathcal{F}) \neq \mathcal{F}^{\mathrm{R}}$ and $\pi^{-1}\left(\mathcal{F}^{\mathrm{R}}\right) \supset \mathcal{F}^{\mathrm{R}}$ strictly, then $\pi$ is (generically) finite on $\mathcal{F}$ and there is a point in $\pi^{-1}\left(\mathcal{F}^{\mathrm{R}}\right) \backslash \mathcal{F}^{\mathrm{R}}$ such that the corresponding K3 surface $S^{\prime}$ admits a rational dominant map

$$
S^{\prime} \rightarrow S=\Phi_{E_{3}}(S) \subset G(3,7)
$$

induced by $\pi$. Indeed, since $\mathbb{C}(\mathcal{F}) / \mathbb{C}(\pi(\mathcal{F}))$ is a finite field extension generated by one primitive element, there exists $\lambda^{\prime} \in \bigwedge^{3} H^{0}\left(S, E_{3}\right)$ (resp. $\left.\sigma_{1}^{\prime}, \sigma_{2}^{\prime}, \sigma_{3}^{\prime} \in \bigwedge^{2} H^{0}\left(S, E_{3}\right)\right)$ such that $S^{\prime}$ coincides with Zariski closure of the locus

$$
G(3,14) \cap \Lambda \cap\left(\left(\lambda-\lambda^{\prime}\right)=\left(\sigma_{1}-\sigma_{1}^{\prime}\right)=\left(\sigma_{2}-\sigma_{2}^{\prime}\right)=\left(\sigma_{3}-\sigma_{3}^{\prime}\right)=0\right) \cap U
$$

(cf. Lemma 3.10).

The restriction $\left.\pi\right|_{S^{\prime}}$ is given by some linear subsystem in $\left|\mathcal{O}_{S^{\prime}}(1)\right|$. Now, composing $\left.\pi\right|_{S^{\prime}}$ with embedding $S=\Phi_{\tilde{E}_{3}}(S) \subset G(3,14)$, we get a rational projection $S^{\prime} \rightarrow S$ between two K3 in $\mathbb{P}^{33}$. Hence (non-degenerate) $S^{\prime}$ and $S$ differ by some projective automorphism. It is then immediate that $S^{\prime}$ belongs to the family $S / \mathcal{F}^{\mathrm{R}}$ and this is a contradiction. 
Thus, under the assumption that $\pi(\mathcal{F}) \neq \mathcal{F}^{\mathrm{R}}$, the map $\pi$ must be birational on $\mathcal{F}\left(\right.$ with $\left.\pi^{-1}\left(\mathcal{F}^{\mathrm{R}}\right)=\mathcal{F}^{\mathrm{R}}\right)$. In this case, the above arguments show that generic surface $S^{\prime}:=S_{64}$ projects birationally onto a K3 of genus 12 (cf. Remark 2.15), which is obviously impossible.

We conclude that $\pi(\mathcal{F})=\mathcal{F}^{\mathrm{R}}$ is the only option.

One may assume w.l. o.g. that $S_{64} \subset \Lambda$ for $S_{64} \subset \mathbb{P}^{33} \supset S$ and $\Lambda \subset H^{0}\left(G(3,14), \bigwedge^{3} \mathcal{E}_{14}\right)$ has codimension 21 . Note also that $\bigwedge^{2} H^{0}\left(S, \widetilde{E}_{3}\right)$ generates a submodule $\mathcal{O}_{S}^{\oplus} \subseteq \bigwedge^{2} \widetilde{E}_{3}$, some $N>1$, which extends to a vector bundle on $\mathcal{F}$. Hence there exist global sections

$$
\widehat{\lambda} \in H^{0}\left(G(3,14), \bigwedge^{3} \mathcal{E}_{14}\right)
$$

and

$$
\widehat{\sigma}_{1}, \widehat{\sigma}_{2}, \widehat{\sigma}_{3} \in H^{0}\left(G(3,14), \bigwedge^{2} \mathcal{E}_{14}\right)
$$

such that $S_{64}$ is contained in the Zariski closure of the locus $\widehat{S}_{64} \cap U$, where

$$
\widehat{S}_{64}:=G_{\Lambda} \cap\left(\widehat{\lambda}=\widehat{\sigma}_{1}=\widehat{\sigma}_{2}=\widehat{\sigma}_{3}=0\right)
$$

(compare with Lemma 3.10).

The family $S_{64} / \mathcal{F}$ extends to an algebraic family $\widehat{S}_{64} / \widehat{\mathcal{F}} \supseteq S_{64} / \mathcal{F}$ (with $\widehat{\mathcal{F}} \supseteq \mathcal{F}$ and conventions on the notation as earlier).

Lemma 4.6. Universal fibration $\widehat{S}_{64} / \widehat{\mathcal{F}} \longrightarrow \widehat{\mathcal{F}}$ is flat at the general point.

Proof. Cutting with hyperplanes we reduce to the 2 -dimensional case. Thus suppose there is a smooth projective surface $V$ and an algebraic family of schemes $Z_{t} \subset V$. One may assume the 0 -dimensional part of all $Z_{t}$ to have the same length and local multiplicities. Then, since the Hilbert polynomial (of a curve) has only $\mathbb{Z}$ - coefficients, we obtain that the 1 - dimensional part of $Z_{t}$ varies algebraically with $t$. Summing up, all $Z_{t}$ have the same Hilbert polynomial, and the claim follows.

According to Lemma 4.6 we may assume the family $\widehat{S}_{64} / \widehat{\mathcal{F}}$ to be flat (for taking the flat closure still leaves $S_{64} / \mathcal{F}$ as a (flat) subfamily). One can also restrict to $\widehat{\mathcal{F}}=\mathcal{F}$ and identify $\mathcal{F}$ with $\mathcal{K}_{33}$ via the Luna's slice theorem (for $S, S_{64}$, etc. being defined up to $\operatorname{Aut} G(3,14)=P G L(14, \mathbb{C})$ ).

Lemma 4.7. There exists, same for all K3 surfaces in $S_{64} / \mathcal{F}$, Zariski open subset $U_{0} \subset G(3,14)$ such that $\widehat{S} \cap U_{0}=S \cap U_{0}$ and $\widehat{S}_{64} \cap U_{0}=S_{64} \cap U_{0}$.

Proof. Flatness and Remark 4.3 imply that all irreducible components of $\widehat{S}_{64} / \mathcal{F}$ are either (contained in) various $G_{\Lambda} \cap I_{\alpha}$, or coincide with some $\left(\mathbb{P}^{1}\right)^{m}$ - bundles over $S_{64} / \mathcal{F}, m \leq 2$. In particular, the dimension of these components is at most

$$
\max \{20,19+2+2\}=23 \text {. }
$$

Further, all irreducible components of $\widehat{S}_{64}$ (resp. $\widehat{S}$ ) different from $S_{64}$ (resp. $S$ ) vary in a flat subfamily, since these residual members have the same Hilbert polynomial.

Thus all these members sweep out a proper subset in $G(3,14)$ of dimension $\leqslant 23$. Removing this yields the needed $U_{0}$.

It follows from Lemma 4.5 that one can write $\widehat{\lambda}=\lambda+\lambda^{0}$ with $\lambda^{0}$ being parameterized by an algebraic curve $Z$ (over $\left.\mathbb{C}\left(\mathcal{F}^{\mathrm{R}}\right)\right)$. Similarly, one has $\widehat{\sigma}_{i}=\sigma_{i}+\sigma_{i}^{0}$, with $Z$-parameterized $\sigma_{i}^{0}$.

Fix some $\lambda^{\prime} \in H^{0}\left(G(3,7), \bigwedge^{3} \mathcal{E}_{7}\right), \sigma_{i}^{\prime} \in H^{0}\left(G(3,7), \bigwedge^{2} \mathcal{E}_{7}\right), 1 \leq i \leq 3$, and a map $f: Z \longrightarrow \mathbb{P}^{1}$. Then associate to $S_{64}$ a K3 surface $S_{64}^{\prime} \subset G(3,7)$ of genus 12 given by $\lambda+f(\lambda, z) \lambda^{\prime}=\sigma_{1}+f(\lambda, z) \sigma_{1}^{\prime}=\ldots=0$ as usual (for $(\lambda, z) \in Z$ corresponding to $\left.S_{64}\right)$. This and Lemma 4.7 deliver a rational dominant map $\phi: \mathcal{K}_{33}-\rightarrow \mathcal{K}_{12}$.

Indeed, the fact that $\phi$ is dominant follows from Proposition 2.9 and simple dimension count, whereas the next result shows that

Lemma 4.8. $\phi$ is correctly defined. 
Proof. Note that the collection of $\Lambda, \lambda, \sigma_{i}$ is defined up to the automorphisms in $P G L(14, \mathbb{C})$. Then the family $\mathcal{F}$ and the locus $U_{0}$ from Lemma 4.7 are defined up to $P G L(14, \mathbb{C})$ as well. Hence by the Luna's slice theorem it suffices to show that any $\tau \in P G L(14, \mathbb{C})$, with $\tau(\mathcal{F})=\mathcal{F}$ and $\tau\left(U_{0}\right)=U_{0}$, induces naturally an automorphism on $G(3,7)$ (thus mapping $S_{64}^{\prime} \subset G(3,7)$ to isomorphic surface). But the latter is evident according to Lemma 4.2 and the assumption on $\tau$.

\section{Proposition 4.9. $\phi$ is birational.}

Proof. Note that the restriction of $\phi$ to the hypersurface $\mathcal{K}_{33}^{\mathrm{R}}$ (cf. Remark 2.11) is smooth because by construction $\phi$ induces an isomorphism there on the tangent spaces. In addition, $\left.\phi\right|_{\mathcal{K}_{33}^{R}}$ is one-to-one onto its image, and hence it suffices to show that $\phi^{-1} \phi(S)=S$.

Let $S_{64} \in \phi^{-1} \phi(S)$ be another K3. Then the corresponding surface $S_{64}^{\prime} \in \mathcal{K}_{12}$ has $f(\lambda, z)=0$ (with notation as above). This gives a rational dominant map $S_{64} \rightarrow S=\Phi_{E_{3}}(S) \subset G(3,7)$ induced by the projection $\pi$ : $G(3,14) \rightarrow G(3,7)$. Here the restriction $\left.\pi\right|_{S_{64}}$ is given by some linear subsystem in $\left|\mathcal{O}_{S_{64}}(1)\right|$. Now, composing $\left.\pi\right|_{S_{64}}$ with embedding $S=\Phi_{\tilde{E}_{3}}(S) \subset G(3,14)$, we get a rational projection $S_{64} \rightarrow S$ between two K3 in $\mathbb{P}^{33}$. This is only possible if $S_{64}$ and $S$ were isomorphic (cf. the proof of Lemma 4.5).

Thus $\phi^{-1} \phi(S)=S$ as wanted.

Finally, Theorem 1.1 follows from Lemma 4.8, Proposition 4.9 and Remark 2.15

Remark 4.10. We can run similar arguments as in the proof of Lemma 3.2 for the vector bundle $E_{4}$ on $S$ (cf. Remarks 2.16 and 2.25) to show that $E_{4} \otimes \mathcal{O}_{S}(C)$ is a rigid rank 4 vector bundle such that $c_{1}\left(E_{4} \otimes \mathcal{O}_{S}(C)\right)=H$ and $\chi\left(S, E_{4} \otimes \mathcal{O}_{S}(C)\right)=13$. Then, similarly as in the proof of Theorem 1.2, one obtains

$$
S \simeq \Phi_{E_{4} \otimes \mathcal{O}_{S}(C)}(S) \subset G(4,13) \cap \mathbb{P}^{36}
$$

for $\left.\mathcal{O}_{S}(H) \simeq \mathcal{O}_{G(4,13)}(1)\right|_{S}$ and $S=\Phi_{E_{4} \otimes \mathcal{O}_{S}(C)}(S)$. Now using Remark 2.16 we prove that $S \subset G(4,13) \cap \mathbb{P}^{36}$ coincides with Zariski closure of the locus $\widetilde{S} \backslash \Pi$ inside

$$
\widetilde{S}:=\Lambda^{*} \cap\left(\lambda^{*}=0\right) \cap\left(\sigma_{1}^{*}=\sigma_{2}^{*}=\sigma_{3}^{*}=0\right)
$$

for some $\lambda^{*} \in H^{0}\left(G(4,13), \bigwedge^{4} \mathcal{E}_{13}\right), \sigma_{i}^{*} \in H^{0}\left(G(4,13), \bigwedge^{2} \mathcal{Q}_{13}^{*}\right)$, where $\Pi$ and $\Lambda^{*}$ are fixed projective subspaces in $\mathbb{P}\left(\bigwedge^{4} \mathbb{C}^{13}\right), \mathcal{E}_{13}$ is the universal vector bundle on $G(4,13)$, and $\mathcal{Q}_{13}^{*}$ is the dual of the universal quotient vector bundle on $G(4,13)$. Finally, applying similar arguments as in the proof of Theorem 1.1, one can show that the same holds for any BN general polarized K3 surface of genus 36, what again leads to the unirationality of $\mathcal{K}_{36}$. Let us stress however that the present strategy does not apply directly to study the unirationality of $\mathcal{K}_{21}$ and $\mathcal{K}_{28}$ (cf. Remark 2.11) because in these two cases there is no apparent relation between the rigid vector bundles on the corresponding polarized K3 and those on the genus 12 surfaces (compare with Lemma 3.2).

\section{REFERENCES}

[1] Baily W. L., Borel A. Compactification of arithmetic quotients of bounded symmetric domains. Ann. of Math. V. 84(2). 1966. P. $442-528$.

[2] Bogomolov F., Tschinkel Yu. Density of rational points on elliptic K3 surfaces // Asian Journ. of Math. 2000. V. 4(2). P. 351 368.

[3] Bourbaki N. Elements of mathematics. Algebra I // New York: Springer Verlag. 1990.

[4] Dolgachev I., Kondo S. Moduli spaces of K3 surfaces and complex ball quotients // Birkhäuser: Progress in Mathematics. 2007. V. 260. P. $43-100$.

[5] Friedman R. Algebraic surfaces and holomorphic vector bundles // New York: Springer Verlag. 1998.

[6] Griffiths P., Harris J. Principles of algebraic geometry // New York: John Wiley. 1978. 
[7] Gritsenko V., Hulek K., Sankaran G. K. The Kodaira dimension of the moduli of K3 surfaces // Inv. Math. V. 167. 2007. P. 519 $-567$.

[8] Hartshorne R. Algebraic geometry // New York: Springer Verlag. 1977.

[9] Iskovskikh V. A., Prokhorov Yu. G. Fano varieties. Encyclopaedia of Mathematical Sciences // Algebraic geometry V / Parshin A. N., Shafarevich I. R. eds. V. 47. Berlin: Springer Verlag. 1999.

[10] Karzhemanov I. Fano threefolds with canonical Gorenstein singularities and big degree // Math. Ann. V. 362 . 2015. P. 1107 1142 .

[11] Karzhemanov I. On Fano threefolds with canonical Gorenstein singularities // Russ. Acad. Sci. Sb. Math. 2009. V. 200(8). P. 111 $-146$.

[12] Karzhemanov I. Remark on polarized K3 surfaces of genus 36 // Geometriae Dedicata. 2012. V. 159(1). P. 295 - 305.

[13] Kondo S. On the Kodaira dimension of the moduli spaces of K3 surfaces // Compositio Math. V. 89. 1993. P. 251 - 299.

[14] Kondo S. On the Kodaira dimension of the moduli spaces of K3 surfaces II // Compositio Math. V. 116. 1999. P. 111 - 117.

[15] Mukai S. Biregular classification of Fano threefolds and Fano manifolds of coindex 3 // Proc. Natl. Acad. Sci. USA. V. 86.1989. P. $3000-3002$.

[16] Mukai S. Curves and K3 surfaces of genus $11 / /$ Moduli of vector bundles (M. Maruyama, ed., eds.). Lecture Notes in Pure and Appl. Math. V. 179. 1996. P. $189-197$.

[17] Mukai S. Curves, K3 surfaces and Fano 3-folds of genus $\leqslant 10 / /$ Algebraic geometry and commutative algebra in honor of M. Nagata. Kinokuniya. 1987. P. $357-377$.

[18] Mukai S. Fano 3-folds // London Math. Soc. Lect. Note Ser. V. 179. 1992. P. 255 - 263.

[19] Mukai S. New developments of Fano varieties: vector bundles and moduli problems // Sugaku. 1995. V. $47(2)$. P. 125 - 144.

[20] Mukai S. On the moduli space of bundles on K3 surfaces I // In "Vector Bundles on Algebraic Varieties ". Tata Institute of Fundamental Research. Bombay. 1987. P. $341-413$.

[21] Mukai S. Polarized K3 surfaces of genus 18 and 20 // Vector bundles and Special Projective Embeddings. Bergen. 1989. P. $264-$ 276.

[22] Mukai S. Polarized K3 surfaces of genus thirteen // Advanced Studies in Pure Mathematics. 2006. V. 45. P. 315 - 326.

[23] Mukai S. Symplectic structure of the moduli space of sheaves on an abelian or K3 surface // Invent. Math. 1984. V. 77 . P. 101 116.

[24] Nikulin V. V. Integral symmetric bilinear forms and some of their geometric applications // Math. USSR Izv. 1979. V. 14. P. 103 $-167$.

[25] Nikulin V. V. Finite automorphism groups of Kähler K3 surfaces // Trans. Moscow Math. Soc. 1980. V. 38. P. 71 - 135.

[26] Saint-Donat B. Projective models of K3 surfaces // American Journal of Mathematics. 1974. V. 96(4). P. 602 - 639.

[27] Viehweg E. Quasi - Projective moduli for polarized manifolds // Ergebnisse der Mathematik. Springer Verlag. V. 30. 1995. P. 1 320.

Courant Institute, NYU, 251 Mercer str., New York, NY 10012, USA

E-mail address: KARZHEMA@CIMS.NYU.EDU 